PALAVRAS. Revista de Epistemología, Metodologia y Ética del Psicoanálisis

ISSN: 2468-9831

www.revistas.unlp.edu.ar/palavras
palavras@outlook.com.ar
Argentina

\title{
EL SIGNO DE FREUD: DIAGNÓSTICO DIFERENCIAL ENTRE PATOLOGÍA ORGÁNICA Y PSÍQUICA
}

DOI $10.24215 / 24689831 \mathrm{e} 015$

Juan de la Cruz Argañaraz

\begin{abstract}
Resumen

The work addresses the problem of the differential El trabajo aborda el problema del diagnóstico diagnosis between organic and psychic pathology. diferencial entre patología orgánica y psíquica. For this it takes as a canon what it calls 'the sign Para ello toma como canon lo que denomina 'el of Freud', making explicit by the Freudian signo de Freud', explicitado a partir de la description of the clinical phenomenon of descripción freudiana del fenómeno clínico de la 'resistance to remembrance'. Expanding the 'resistencia a la rememoración'. Expandiendo la structure of this 'canon' it is proposed to elucidate estructura de este 'canon' se propone elucidar la the subjective implication with the symptom, as a implicación subjetiva con el sintoma, como central element for the differential diagnosis elemento central para el diagnóstico diferencial between organic and psychic pathology. One of entre patología orgánica y psíquica. Se analiza these forms of subjective involvement is analyzed, luego una de estas formas de implicación not described by psychoanalysis but by the subjetiva, no descripta por el psicoanálisis sino clinicians of the 19th century: 'la belle indifférence por los clinicos del S. XIX: "la belle indifférence des des hysteriques. Thus, the crucial point for the hysteriques. Así, el punto crucial para el diagnosis of conversion hysteria at the beginning diagnóstico de la histeria de conversión en el inicio of Psychoanalysis and its advances in the del Psicoanálisis y sus avances en la delimitación delimitation of Psychopathology came from clinical de la Psicopatologia provino de descripciones descriptions that were explained and theorized by clinicas que fueron explicadas y teorizadas por êl. him. In this sense, the importance of the 'sign of En este sentido, la importancia del 'signo de Freud' Freud' is that it is a sign of clinical listening that es que es un signo de la escucha clinica utilizable can be used in any clinical examination and does en cualquier examen clínico y que no requiere not require the assumption of certain theories of suponer ciertas las teorias del Psicoanálisis. El Psychoanalysis. The work exposes casuistry trabajo expone casuistica discutiendo la discussing the fecundity of this perspective and fecundidad de esta perspectiva $y$ algunas some current consequences of the abandonment of consecuencias actuales del abandono de la clinical listening. escucha clinica.
\end{abstract}

Key words: Psychoanalysis, Psychopathology, Palabras claves: Psicoanálisis, Psicopatologia, Differential Diagnosis, Epistemology.

Diagnóstico Diferencial, Epistemología.

\section{Cómo citar este artículo:}

De la Cruz Argañaraz, J. (2017). El Signo de Freud: diagnóstico diferencial entre patología orgánica y psíquica. Palauras. Revista de Epistemología, Metodología y Ética del Psicoanálisis, 3,1-14. Recuperado de www.revistas.unlp.edu.ar/palavras 


\section{EL SIGNO DE FREUD: DIAGNÓSTICO DIFERENCIAL ENTRE PATOLOGÍA ORGÁNICA Y PSÍQUICA}

Juan de la Cruz Argañaraz ${ }^{1 \star}$

“...nada facilita tanto la observación clínica como la sintomatología completa y precisa. Más allá de los procesos mentales, catalogados por la psicologia actual, hay un mundo entero de detalles psíquicos, los cuales, desde el punto de vista psicológico todavia son tierra desconocida y solo pueden ser descubiertos mediante una laboriosa investigación, aunque están contenidos en los fenómenos psíquicos generales, suceden ante los ojos de todo el mundoy pueden ser encontrados esparcidos como insinuaciones en escritos en donde uno menos los buscaria, particularmente en la obra de dramaturgos $y$ de los novelistas [...] Aqui es necesario encontrar el camino en el laberinto de las manifestaciones." (Kalhbaum, 1874/1996: 114).

\section{Introducción}

Este párrafo, de uno de los más grandes clínicos que haya producido la Psiquiatría decimonónicá ${ }^{2}$, no deja de producirnos, leído desde la actualidad, cierta piedad trágica hacia su autor. Kalhbaum parece intuir oscuramente, en 1874, ese universo de lapsus linguae, recuerdos encubridores lapsus calami, de olvidos y actos sintomáticos que Freud expone treinta años después mostrando que, efectivamente, estos fenómenos estaban presentes en Shakespeare o Goethe. Al parecer, la alienación de la Psiquiatria decimonónica al campo de la mirada descripta por Foucault en su (1966), invalida al maestro clinico a tal punto, que confunde sus sentidos y busca con 'los ojos' lo que intuye que está en el registro de lo escrito -'dramaturgos y novelistas' -. Es decir que, de algún modo, el pasaje de la clínica de la mirada a la clínica de la escucha fundada por Freud, quizá tiene aquí, junto con Breuer, otro de sus agonistas.

\footnotetext{
1* Facultad de Psicologia de la Universidad Nacional de Córdoba, Argentina. Mail: juanarga@.gmail.com

${ }^{2}$ Karl Kalhbaum describe y establece la Catatonía que nunca más fue cuestionada hasta la actualidad, orientando y proveyendo a su discipulo Hecker la casuística para describir y establecer la Hebefrenia.
} 
La via di levare que instaura la escucha, con el 'método de la asociación libre', permite a Freud abrir un universo de fenómenos a su vez desconocidos y extrañamente consabidos. Sin embargo, el dominio de la clinica por el campo de la mirada mantiene aún hoy su hegemonía dificultando la transmisión de qué es un hecho clínico. Cabe realizar sobre esto la misma reflexión que adelanta L. Fleck $(1935,1986)$ para el análisis epistemológico de la 'reacción de Wassermann' (serologia de la sifilis). Dice Fleck:

Reflexioné durante bastante tiempo sobre cómo podria exponer la reacción de Wassermann a alguien que no fuera especialista, pero ninguna descripción puede sustituir a la idea que se adquiere después de muchos años de experiencia práctica con la reacción. (...) La reacción tiene un esquema fijo, pero se ejecuta con tantas modificaciones como laboratorios existen. Se basa en cálculos cuantitativos exactos; sin embargo, el 'ojo clínico' -que en este caso podria llamarse 'intuición serológica'- es mucho más importante que el cálculo. (Fleck, L. 1935/1986: 100).

La misma dificultad enfrentamos al exponer, hechos 'evidentes' para el diagnóstico en psicopatología clínica. El mismo término 'evidencia' está determinado por la hegemonia de la mirada y lo obvio a la vista. $\mathrm{Al}$ epistemólogo le puede parecer completamente 'arbitrario', por ejemplo, el diagnóstico de 'mania' que hará el psicoanalista o el clínico, a un sujeto que solo parece 'feliz', 'alegre' y eufórico. Sin embargo, aún con enormes diferencias teóricas, en la 'comunidad de clínicos', no resulta una dificultad acordar o debatir por qué es adecuado, o no, ese diagnóstico. Hasta hace pocos años la incomunicabilidad nunca llegó a tales extremos, aunque actualmente se corra ese riesgo.

Con estas dificultades de transmisión presentes, intentamos el abordaje de un punto que siempre ha sido objeto de controversia en el diagnóstico psicopatológico, y que está vinculado a la permanente hegemonía psiquiátrica en el área. Este es, la supuesta insuficiencia de la formación de psicólogos y psicoanalistas no médicos para el diagnóstico diferencial entre patología de origen psíquico y orgánico. A esa controversia se orienta este trabajo, en parte explicitando y en parte teorizando los 'modelos' que Freud 
legó de su escucha clinica, y algunos elementos frecuentemente usados en la práctica del psicoanálisis de modo 'informal'. Veremos que, en verdad, este tópico es un punto fundacional del Psicoanálisis y la Psicopatología.

Dice Freud en 1904, escribiendo en tercera persona:

Sin amnesia de alguna clase no existe historial clínico neurótico. Si se insta al relator a llenar estas lagunas de su memoria mediante un esforzado trabajo de atención, se advierte que las ocurrencias que le vienen sobre este punto son refrenadas \{zurückdränger) por él con todos los recursos de la critica, hasta que por fin siente un franco malestar cuando se le instala realmente el recuerdo. De esta experiencia, Freud infirió que las amnesias son el resultado de un proceso que él llama represión (verdrängung (...) las fuerzas psiquicas que han originado esta represión, cree registrarlas en la resistencia que se opone a la reproducción..." (Freud, 1904/1996: 239).

En este pảrrafo Freud presenta la "resistencia a la rememoración" cuando se insta al paciente a la recuperación del recuerdo por un trabajo psíquico. Esta maniobra clínica que Freud utiliza con otros objetivos, puede legitimamente denominarse como signo de Freud. Recuérdese que, clásicamente, la clínica médica ha diferenciado entresintoma (en sentido pre- psicoanalítico) y signo clinico. El síntoma, es relatado por el paciente y tiene carácter subjetivo, se manifiesta directamente al examen o al interrogatorio. Por el contrario, elsigno tiene un carácter objetivo y requiere una maniobra o acción premeditada del médico. Ejemplos simples e ilustrativos de la importancia de estos signos clínicos pueden ser epulso paradojal o signo de Griesinger, en el cual se le solicita al paciente una inspiración profunda mientras se le ausculta. Si hay una infección del pericardio el ritmo cardiaco es el opuesto al esperado. En elsigno de Babinsky, el examinador procede a pasar un objeto, su dedo o una lapicera, longitudinalmente por la planta del pie del recién nacido: si no contrae los dedos del pie y/o la pierna, hay signo de Babinsky, que indica daño neuronal por ausencia del reflejo homónimo. Repitamos la cita anterior que muestra el procedimiento del signo de Freud: 
1- Si se insta al relator a llenar estas lagunas de su memoria mediante un esforzado trabajo de atención, (maniobra clínica)

2- Se advierte que las ocurrencias que le vienen sobre este punto son refrenadas \{zurückdrängen\} por él con todos los recursos de la critica, (implicación subjetiva)

3- Hasta que por fin siente un franco malestar cuando se le instala realmente el recuerdo (reacción paradojal a la restitución de una función afectada).

Este signo de Freud, podria utilizarse clinicamente en el diagnóstico diferencial de las amnesias pre-seniles o seniles de origen psíquico $u$ orgánico. Luego de:

1- instar a un esforzado trabajo de atención para llenar las lagunas de la memoria, (maniobra clinica);

2- el amnésico orgánico no refrena ni critica las ocurrencias, (predispuesto a colaborar en el trabajo, da la información al clinico) y

3- cuando el recuerdo se instala nuevamente en la conciencia, la reacción de un amnésico de origen orgánico es inversa que en el neurótico: no se presenta un franco malestar, sino una reacción de asombro $y$ júbilo por el recuerdo recuperado y la función restituida. $^{3}$

Aunque al igual que todos los otros signos, siempre son considerados en el marco de la evaluación clinica global, aquí tenemos un modelo del modo de utilizar clínicamente este esquema. Laresistencia y el franco malestar son los indicios del alcance y dimensión de la implicación subjetiva del paciente en el sintoma. Es crucial subrayar que tal maniobra es imposible si no se ha abandonado la hipnosis, es decir, que estesigno de Freud es resultado del

\footnotetext{
${ }^{3}$ Luego el paciente puede, obviamente, preocuparse por tomar conciencia de su disfunción.
} 
pasaje del método de Breuer al método de la asociación libre y el cambio correlativo en la posición de Freud. Es crucial porque, efectivamente, en la hipnosis el sujeto $\mathrm{y}$ su implicación subjetiva con el sintoma queda supeditada a la sugestión del otro. Tomando como 'canon' el signo de Freud para el diagnóstico diferencial de amnesias, intentaremos expandir su aplicación a otros casos clínicos.

\section{Implicación subjetiva y diagnóstico diferencial}

Este trabajo tiene un antecedente mediato en una amigable discusión que, hace ya más de veinte años, tuvimos con una Pediatra acerca del diagnóstico diferencial entre patologias de origen orgánico y psíquico. Según ella, los psicoanalistas no médicos no estaban en condiciones de realizar dicho diagnóstico diferencial y podian tratar duranteaños a un paciente cuyo sintoma tuviese origen orgánico. Habiamos sostenido en aquel momento que el diagnóstico diferencial podía realizarse por la escucha, ya que el discurso del paciente y su implicación subjetiva con el fenómeno patológico, era completamente diverso en cada una de esas situaciones. Tiempo después, esta Pediatra nos comenta el siguiente recorte de su práctica clínica pediátrica:

"El otro día me acordé de vos. Recibi por primera vez en años un caso de enuresis orgánica. Realmente la presentación era completamente diferente a todas las demás. Las consultas de enuresis son muy frecuentes y siempre se presentan más o menos igual: a la consulta viene la madre - el padre no aparece -, y entra al consultorio hablando del niño, que generalmente no me mira ni se dirige a mí. El niño se mantiene pegado al cuerpo de la madre, expectante, avergonzado, con la mirada esquiva y sin prestarse al interrogatorio que siempre responde la madre. Con variaciones ese es el molde de todas las consultas por enuresis y son derivadas al área de salud mental. El otro día, abro la puerta del consultorio y entra un niño de alrededor de 10 años, atrás, sus dos padres. Se sientan y el niño me dice: 'Me meo. No sé qué me pasa. Estoy en cualquier parte, jugando a la pelota, en la escuela y me meo. No me doy cuenta y se me cae el pis por el pantalón.' Me sorprendió muchísimo y, efectivamente, tenía una pequeña tumoración benigna calcificada en el esfinter de la vejiga." 
La sorpresa de esta pediatra se produce justamente, porque la enorme mayoria de los casos de enuresis infantil, son sintomas de origen psíquico donde el retorno de lo reprimido deja al niño inevitablemente implicado en su sintoma. Manifiesta resistencia al examen clinico, responde con monosílabos y retacea información de su padecer donde está concernido. El niño se comporta como el neurótico al cual Freud insta a cubrir las lagunas de su memoria. Por el contrario, en este caso de origen orgánico, el niño registra el sintoma, la enuresis, como un evento externo a su subjetividad, algo que le acontece a su cuerpo y su discurso se dirige a entregar toda la información necesaria para eliminarlo. Se presta al interrogatorio, enfrenta y demanda una solución al médico en la consulta.

En este mismo sentido, la vía del Psicoanálisis resulta, en general, la interrogación de la implicación subjetiva. En Delirio: historia, clínica, metateoria, Germán Berrios (1996) ha expuesto, desde la Psiquiatria, las diversas perspectivas que a través de la historia se han presentado para poder diferenciar de un modo claro y distinto qué es un delirio y qué no. Los abordajes lógicos, de contenido, retóricos y de consistencia, no terminan de lograr el discernimiento en todos los casos de algún elemento objetivo de la estructura de un delirio. En la misma linea que venimos exponiendo, Jacques Lacan apunta al sujeto de la enunciación y ha expuesto un elemento central que es la certeza como indicio de psicosis (1955-1956/1986), la cual también indica la implicación subjetiva sobre el enunciado para considerarlo delirante. Es decir que, aunque se trate de un enunciado aparentemente científico, religioso o de la exposición de una teoria de cualquier área del conocimiento, laimplicación subjetivacon estos enunciados, al grado de la certeza, es un indicador que el Psicoanálisis podria desarrollar como linea de investigación, diferenciando la certeza del convencimiento, la creencia o la convicción. Claro está que este modo de abordaje, se articula fácilmente al Psicoanálisis en tanto este sostiene una posición ética, cada vez más extraña al discurso médico psiquiátrico en el paso del siglo XX al XXI, que no solo rechaza al sujeto sino hasta su filiación a la antigua tradición de la clínica, que el Psicoanálisis mantiene viva: 
Se trata de que la clínica y la ética, comparten un mismo método. Esto puede parecer extraño cuando se oye por primera vez, pero deja de serlo en cuanto se reflexiona sobre ello con cierta calma. Hay un hecho que no deja de ser sorprendente, y es que el método de la ética procede con toda probabilidad del método de la clínica, más concretamente del método de la clínica hipocrática. El autor de esa modificación fue Aristóteles. Hijo de médico, muy preocupado siempre por las cuestiones médicas e investigador incansable de problemas biológicos, Aristóteles llegó muy probablemente a la ética desde la medicina. Cuando en la Ética a Nicómaco describe la lógica del razonamiento práctico, no está pensando sólo en la ética y la política sino también, como él mismo se encarga de señalar, en la técnica, especialmente en aquella que en su época se habia convertido en paradigmática, la téchne iatriké, la técnica médica, la medicina. Toda la teoria de la deliberación, la prudencia, el término medio, el razonamiento probable, la toma de decisiones en situación de incertidumbre, etc., se aplica por igual a la clínica y a la ética." (García, 2001: 19).

En verdad, debiera resultar obvio por qué la escucha psicoanalitica se estatuye en una herramienta clinica poderosa para el diagnóstico diferencial. Un modelo clínico de esta vinculación entre el sujeto y su síntoma, fue inaugural al Psicoanálisis y presentado tempranamente por Freud en Estudios sobre la Histeria

Efectivamente, el diagnóstico diferencial entre sintomas orgánicos y psíquicos se hizo consustancial al Psicoanálisis mostrando su progresividad (Argañaraz, 2004) respecto a la Psiquiatria clínica decimonónica, por responder un enigma largamente sufrido por ella. El Psicoanálisis se fundó en la escucha de la histeria de conversión, patologia en la cual el diagnóstico diferencial entre lo orgánico y psíquico era el centro del debate clínico. La característica "patoplastia" -es decir la plasticidad de sus manifestaciones patológicas, que pueden tomar cualquier forma de enfermedad orgánica desde la ceguera hasta la epilepsia-, fue el vía crucis del diagnóstico del psiquiatra y el médico clínico del siglo XIX. Al establecer su etiología psíquica, el Psicoanálisis también hecha las bases de la psico-patologia, término poco presente en el área hasta laPsicopatología de la vida cotidiana En la Histeria de conversión, la implicación subjetiva produce un fenómeno 
reconocido desde Charcot (1888/2007), pero que solo fue explicado por la teoria psicoanalítica de la represión:

...un colega de mi amistad me pidió que examinase a una joven dama que desde hacia más de dos años padecía de dolores en las piernas y caminaba mal.. (....) Parecía inteligente y psíquicamente normal, y sobrellevaba con espíritu alegre su padecer, que le enervaba todo trato y todo goce; lo sobrellevaba con la "belle indifférence de los histéricos, no pude menos que pensar yo. (Freud, 1895/1996: 151).

\title{
De la "belle indifférence" hacia el sintoma, a la posición subjetiva
}

Esa belle indifférence es explicada por la teoria de "La represión". Dice Freud en el texto correspondiente de la Metapsicologia:

\begin{abstract}
A una apreciación por entero diversa del proceso represivo nos fuerza el cuadro de la genuina histeria de conversión. Lo sobresaliente en ella es que consigue hacer desaparecer por completo el monto de afecto. El enfermo exhibe entonces hacia sus sintomas la conducta que Charcot ha llamadoula belle indifférence des hystériques" (...) La represión de la histeria (de conversión) puede juzgarse totalmente fracasada en la medida en que solo se ha vuelto posible mediante unas extensas formaciones sustitutivas; pero con respecto a la finiquitación del monto de afecto, que es la genuina tarea de la represión, por regla general constituye un éxito completo. (Freud, 1915/1996: 150-151).
\end{abstract}

Al igual que en Freud, en Jacques Lacan se presenta tempranamente, el análisis de la posición subjetiva de la histeria de conversión, vinculando la posición de ella con 'el alma bella' hegeliana en su puntuación de 1951 del "Análisis fragmentario de un caso de histeria (el caso Dora)". En base a la 'bella indiferencia' como indicador consensuado en la comunidad clínica, Freud desarrolla la explicación del fenómeno en su teoría de la represión dando cuenta de la implicación del sujeto con su sintoma. Con ello, le es posible a Lacan desplegar el análisis estructural de laposición subjetivade la Histeria de conversión. Es decir que la 'bella indiferencia' hacia el síntoma, es correlativa de la posición subjetivadel 'alma bella'. Esta posición es la que 
hace refractaria a la Histeria de conversión al dispositivo médico-psiquiátrico, en tanto éste conlleva un lugar del Otro de dominio y domesticación. De ahí que Lacan subraye el lugar en el que es colocado Freud y el cambio de posición del psicoanalista respecto al médico-psiquiatra para generar un cambio de posición subjetiva. Como lo desarrolla Lacan en "Intervención sobre la transferencia", Freud se encuentra frente a Dora:

...colocado frente a la pregunta, por lo demás de un tipo clásico en los comienzos del tratamiento: 'Esos hechos están ahí, proceden de la realidad y no de mí, ¿Qué quiere usted cambiar en ellos?' A lo cual Freud responde por:

Una primera inversión dialéctica que no tiene nada que envidiar al análisis hegeliano de la reivindicación del "alma bella" la que se rebela contra el mundo en nombre de la ley del corazón: "mira, le dice, cuál es tu propia parte en el desorden del que te quejas'. Y aparece entonces:

Un segundo desarrollo de la verdad a saber, que no es sólo por el silencio, sino gracias a la complicidad de Dora misma, más aun: bajo su protección vigilante, como pudo durar la ficción que permitió prolongarse a la relación de los dos amantes. (Lacan, 1951/2009: 213; cursivas del original, subrayado añadido).

Con lo expuesto analicemos otro recorte clinico :

Una paciente de 58 años es enviada a tratamiento psicológico por su médico, bajo sospecha de un sintoma de conversión histérica. La paciente había sufrido numerosos avatares, mala praxis médica con riesgo de invalidez, intervención de abogados y graves problemas con su obra social durante años, hasta que finalmente es operada de una cadera y se le coloca una prótesis. La paciente se recupera favorablemente pero comienza a renguear. Se queja de molestias difusas y los estudios médicos indican que no existe ninguna razón para el rengueo y las molestias. Dado lo psicológicamente traumático del proceso de su tratamiento, y la enorme gravitación que habia ejercido en su vida cotidiana por más de cinco años, el médico sospecha una histeria de conversión. Ella accede a consultar porque el médico se le presenta la derivación como un 'apoyo psicológico'. Durante las tres primeras entrevistas, la paciente relata sus molestias, su dificultad para caminar, los distintos estudios que ha realizado y sigue realizando para encontrar la causa. Expone largamente a la más mínima pregunta o solicitud de aclaración sobre algún detalle. Cuenta la larga historia

\footnotetext{
${ }^{4}$ Debo los siguiente dos casos a la Dra. Maria Leonor Argañaraz, psicoanalista de origen
} médico. 
de avatares de su enfermedad, la mala praxis sufrida, los miedos de quedar semi-inválida. Entrevista tras entrevista, la paciente no habla de otra cosa. Cuenta cuál es la próxima consulta que va a realizar, los trámites en la obra social y su entusiasmo por poder reincorporarse a su trabajo a pesar de las dificultades al caminar. Solicito que realice una consulta con un infectólogo de mi confianza. En la quinta entrevista narra, aliviada, que sufre una poco frecuente infección ósea intrahospitalaria, que no había sido detectada y probablemente contagiada durante las varias horas que estuvo en quirófano por su cadera. Esto explica sus difusas molestias y su rengueo.

Un proceso represivo nunca permite el fluir espontáneo de la consulta sobre un tópico afectado por este proceso. A pesar de haber sufrido mala praxis médica - que facilitaría posicionarse como 'alma bella' - nada de esa posición subjetiva de la Histeria de conversión en esta paciente: no se queja del desorden del mundo sino que trabaja para resolver sus problemas. Nada de "belle indifférence" hacia el sintoma. Si bien es elogiable la amplitud de criterio del médico tratante que realiza la derivación a la psicoanalista, resultaria preferible que profundizara su tarea de diagnóstico clínico considerando lo que escucha en la paciente, al igual que el niño de la enuresis orgánica: que se atienda su reclamo de curar su padecer. Otro caso de simple y fundamental "escucha clínica":

Una mujer de un poco menos de 50 años está internada en el servicio de Clínica Medica, su sintoma es una abulia absoluta. Permanece todo el día en cama, hay que exigirle que se alimente, no le interesa hacer nada. Cuando se la interroga habla en voz baja y con una lentitud insoportable, no hay signos evidentes de ninguna enfermedad física (fiebre, hipertensión, etc.) El examen físico no arroja ningún resultado orientador, los estudios básicos (análisis de rutina, radiografia de tórax, etc.) aparecen normales. Se decide derivarla al Hospital Neuropsiquiatrico con la sospecha de cuadro de Melancolía. Antes de la derivación realizo una minuciosa historia clínica aunque la paciente ya tenía su historia clinica. Ante mis preguntas acerca de su estado, ella solo responde que "no tiene ánimo," que su familia se enoja pero ella no tiene ánimo para nada. Hasta hace un tiempo realizaba las tareas del hogar sin problemas y atendia adecuadamente a su marido y sus siete hijos, considera que ha sido una buena ama de casa y buena madre hasta que comenzó a dejar de tener ánimo; no se reprocha nada, mantiene su autoestima, no ha sufrido ninguna perdida en los 
últimos años. El discurso de esta mujer descarta el cuadro melancólica podemos ignorar que es lo que le sucede, pero sabemos que no está melancólica.

En determinado momento le pregunto por sus embarazos y sus partos, me responde que todos sus embarazos fueron normales y sus siete hijos nacieron por parto natural; le pregunto si ha amamantado a sus niños, me dice que les dio de mamar hasta más o menos los dos años pero que al último no pudo darle el pecho porque no tenía leche (este dato es clave). Pregunto entonces si en el último parto sucedió algo raro y me responde que si, que ella sufrió una gran hemorragia con caída de la tensión arterial y desvanecimiento. Con este dato se completa el diagnostico el cual era obviamente un Panhipopituitarismo o Enfermedad de Simmonds-Sheehan .

No fue derivada. Se inició tratamiento con suplencia hormonal y evoluciono favorablemente saliendo de su apatía. Era el año 1986, el protocolo de Hospital Neuropsiquiátrico de Córdoba establecía el tratamiento con electroshock para todos los casos de Melancolía y algunos casos de Fobias.

No se trata de saber medicina sino de atender al sujeto, de examinar su discurso, de tomar en serio las reglas de la represión, del método de la asociación libre, en fin, de la escucha clínica.

\section{Discusión}

Todo el desarrollo de la nomenclatura de la Clasificación Internacional de Enfermedades (CIE 10) que, influida por el DSM IV y la Asociación de Psiquiatras Americanos, eliminó la Histeria como entidad despedazándola en diversos síndromes, parece formar parte de una estrategia, cientificamente poco honesta, de no confrontar con el Psicoanálisis. Efectivamente, haciendo desaparecer la Histeria, se consigue destruir buena parte de la base empírica del Psicoanálisis, base empírica que tomó de la psiquiatría clínica del siglo XIX demostrando su progreso científico respecto de ella. El riesgo grave de tal deshonestidad es hacer desaparecer también la Psicopatología. Una

\footnotetext{
${ }^{5}$ Lo que sucede es que durante el embarazo la hipófisis pasa a tener un tamaño entre siete y diez veces mayor al que tiene antes del embarazo, pero la arteria que la irriga sigue teniendo del mismo calibre, esto hace que ante una disminución de la irrigación sanguínea esta glándula sufre la isquemia (falta de sangre) mucho más que otros órganos y se necrosa (se muere); por eso esta mujer no pudo amamantar al último hijo ya que al no haber hipófisis no hay prolactina; la hipófisis comanda a otra glándulas de modo que si ella no produce ACTH la suprarrenal no libera corticoides, si ella no produce TSH la tiroides no libera hormona tiroidea, etc.
} 
clínica sindrómica -escandalosamente similar a la doctrina de las monomanías de Esquirol que nos produce una regresión al principio del siglo XIX- diseñada para corresponder punto a punto con distintos psicofármacos, no solo no interroga la implicación subjetiva con el sintoma, sino que elimina lisa y llanamente al sujeto mismo. Eso no impide que la Histeria siga existiendo.

En su "Cómo desembarazarse de la histeria o la histeria en el siglo XX", J.C. Maleval disecciona muchos intentos, desde Babinsky, para desmontar la Histeria como entidad clínica y por supuesto su origen psíquico:

...el austriaco Walcher es alabado porque, en 1969, introdujo la noción de 'depresión enmascarada' también llamada 'depresión somatizada' o, y aún mejor, 'depresión sonriente'. El desconocimiento del sujeto del inconciente no permite distinguir entre las denominaciones paradójicas del sindrome las versiones actuales de la bella indiferencia [...] la muy hipotética 'depresión sonriente' [...] parece destinada a tener futuro, puesto que los laboratorios farmacéuticos lo consideran clinicamente excelente y no regatean esfuerzos para promocionarla. La depresión enmascarada se inscribe a las mil maravillas en la clínica del medicamento..." (Maleval, J. C., 1994: 273).

El futuro, y nuestro trabajo cotidiano, dirá si tales intentos podrán consolidarse.

\section{Referencias bibliográficas}

Argañaraz, J. (2004). "La constitución del Psicoanálisis como 'programa de investigación". En "Epistemologia e Historia de la Ciencia" Selección de trabajos vol. 10. Pío García y Patricia Morey Editores U.N.C.

Berrios, G.; Fuentenebro de Diego, F. (1996). Delirio: historia, clinica, metateoria. Madrid: Trotta.

Breuer, J. \& Freud, S. (1895/1996). "Estudios sobre la histeria". En: S. Freud, Obras Completas Vol. VII Buenos Aires: Amorrortu. 
Charcot, J.M. (1888/2007). "Parálisis hístero -traumática masculina". En: Conti y Stagnaro (Comp.), Historia de la ansiedad Buenos Aires: Polemos.

Fleck, L. (1935/1986). La génesis y el desarrollo de un hecho científico Madrid: alianza.

Foucault M. (1966). El nacimiento de la clinica. México: Siglo XXI.

Freud, S. (1904/1996). “El método psicoanalitico de Freud". En: S. Freud, Obras Completas, Vol. VII. Buenos Aires: Amorrortu.

Freud, S., (1915/1996), “La represión” En: S. Freud,Obras Completas, Vol. XIV. Buenos Aires: Amorrortu.

Garcia, D. (2001). La deliberación moral: el mêtodo de la ética clínica. Medicina Clínica, 1171), 18-23.

Kalhbaum, K. (1874/1996). "La Catatonia o locura de tensión”. En: La locura maniaco - depresiva. La Catatonia. La hebefreniaBuenos Aires: Polemos.

Lacan, J. (1951/2009). “Intervención sobre la transferencia". EnEscritos I. Buenos aires: Paidos.

Lacan, J. (1955-1956/1986). “El fenómeno psicótico y su mecanismo” EnEl Seminario III. Las psicosis Buenos Aires: Paidós.

Maleval, J. C. (1994). Cómo desembarazarse de la histeria o la histeria en el siglo XX. Revista de la Asociación Española de Neuropsiquiatria, 1(\#9), 269-290. 